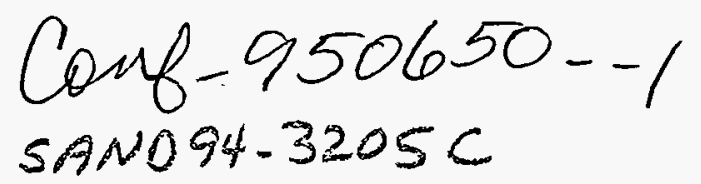

\title{
AN APPLICATION OF MECHANICAL LEVERAGE TO MICROACTUATION
}

\author{
J. J. Sniegowski ${ }^{*}$ and C. Smith ${ }^{* *}$ \\ *Sandia National Laboratories, MS-1080, Albuquerque, NM 87185-1080, USA \\ ** University of Wisconsin - Madison, Madison WI, 53706 USA
}

\begin{abstract}
SUMMARY
Preliminary results on the use of mechanical advantage to convert a short-displacement, high-force actuation mechanism into a long-displacement, medium-force actuator are presented. This micromechanical, mechanically-advantaged actuator is capable of relatively large displacement and force values. The target design values are a lever ratio of 17.5:1 leading to a $\pm 17.5 \mu \mathrm{m}$ displacement while providing no less than $2.25 \mu \mathrm{N}$ of force throughout actuator's range of motion for an applied voltage of less than 50 volts. The basis for the mechanical advantage is simple levers with fulcrums.
\end{abstract}

\section{INTRODUCTION}

A search for suitable and available surfacemicromachined micro-drivers for applications of interest can lead to disappointment. Often one would like to have a high-force $(\geq 1 \mu \mathrm{N})$, large-displacement $(\geq 10 \mu \mathrm{m})$ linear actuation mechanism available. Unfortunately, high-force actuators are typically limited to short displacements $(\leq 2 \mu \mathrm{m})$, while large displacement mechanisms are typically limited to small forces or consume an undesirable amount of chip area. We propose using mechanical levers to magnify highforce, small-displacement actuation, thereby providing medium-force actuation with large displacement. Such a leverage system could be used to increase an actuation force further if desired, but, of course, at the expense of displacement. The use of mechanical advantage, in the form of levers, has been demonstrated before, for example in micromechanical devices designed to analyze film intrinsic strain [1] and to study tensile stress samples [2]. There have also been microactuators which utilize mechanical deformations and the geometry of the deformations $[3,4]$ to obtain the desired motion and force. However, we believe this to be the first device to utilize the classical lever-and-fulcrum approach for microactuation.

To demonstrate the application of this principle to actuation, we chose an electrostatic parallel-plate configuration for its simplicity of fabrication by surface micromachining and ease of connection to a lever system. To first order, the basic construct consists of an array of parallel plate capacitors. The array provides input to a set of mechanical levers that reduce the force by the lever ratio (i.e. in this case, 17.5:1) but magnify the displacement by the same ratio. A synopsis of the electro-mechanical design, including a discussion of the choice of flexure fulcrums, is provided in this paper. Compromise among many issues lead to a primary design, although several other configurations of electrostatic parallel-plate shuttles, levers, and fulcrums are being investigated. The section on design summary provides the arguments that lead to the primary design. Figure 1 is a SEM image of one of the fabricated primary design variations and illustrates the desired principle of operation.

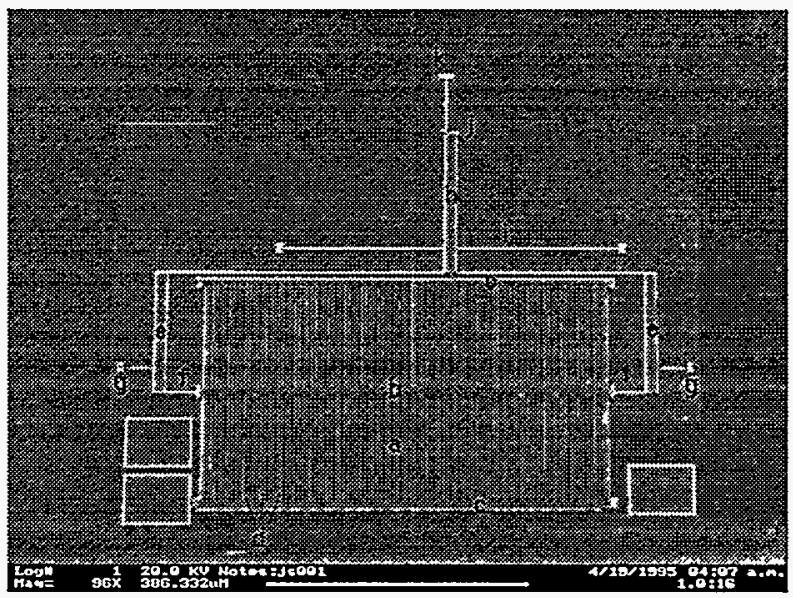

Figure 1. SEM micrograph of a fabricated actuator. The device is fabricated from 2.0- $\mu$ m-thick polysilicon by standard surface micromachining techniques.

In this figure, an array of parallel-plate capacitors is shown at the lower center (a). The array consists of a central spine (b) with fingers extending to both sides. Along the outside edges run two supports (c) which then join to the central spine at the left and right ends. This forms the 'box' configuration. Between each set of shuttle fingers on the movable shuttle is a set of two fixed finger electrodes (d). These electrodes allow the array to be directed either to the left or to the right. The central spine is connected to the first levers (e) by flexible joints (f), shown coming out of the array to levers on both ends. This provides a pull-pull arrangement to the second lever. The first lever is supported at its fulcrum by two flexure elements (g) attached to the substrate. This first lever, in turn, is connected to the second lever $(\mathrm{h})$ by a flex joint. The 


\section{DISCLAIMER}

This report was prepared as an account of work sponsored by an agency of the United States Government. Neither the United States Government nor any agency thereof, nor any of their employees, makes any warranty, express or implied, or assumes any legal liability or responsibility for the accuracy, completeness, or usefulness of any information, apparatus, product, or process disclosed, or represents that its use would not infringe privately owned rights. Reference herein to any specific commercial product, process, or service by trade name, trademark, manufacturer, or otherwise does not necessarily constitute or imply its endorsement, recommendation, or favoring by the United States Government or any agency thereof. The views and opinions of authors expressed herein do not necessarily state or reflect those of the United States Government or any agency thereof. 


\section{DISCLAIMER}

Portions of this document may be illegible in electronic image products. Images are produced from the best available original document. 
fulcrum of the second lever (i) is also a flexure element. The output of the device is produced at the end of the lever (j) at the upper center of the photo. The second lever basically sweeps out a horizontal arc producing left-right motion parallel to the shuttle motion. The output acts against a simple cantilever spring (k) to indicate the force level. This spring is visible at the center top of the SEM.

For the device shown, the parallel array motion is no more than a micron, but is levered to produce \pm 17 $\mu \mathrm{m}$ displacement at the end of the second lever. Motion against a cantilever spring provides a simple force measurement which has been demonstrated with the preliminary devices.

The motivation for designing this mechanical system becomes obvious when one observes the electrostatic comb-driven microengine[5]. Although the microengine operates extremely well with the current choice of electrostatic comb-drive linear actuation, the intent is to reduce the consumed chip area required for the linear actuators or to increase the available linear forces without further consumption of chip area. Initial results indicate that this may be accomplished using the parallel plate and lever configuration. Direct replacement of the comb-drive linear actuators with a leveraged, parallel-plate actuator having the same force and displacement generation reduces consumed chip area by roughly a factor of five.

\section{FABRICATION}

Fabrication of the micro-levered actuator can be accomplished with a single-level, polysilicon surface micromachining process. Single-level implies one level of free-standing, mechanical polysilicon, and, in this process, includes a level of highly conductive polysilicon which serves as both electrical interconnects and voltage reference planes. The device designs reported here are fabricated with a single-level process, however, multiple-level processes[5] are also quite suitable and allow greater flexibility in the design of the fulcrums.

The structures are fabricated on silicon substrates separated from the devices by a composite dielectric layer of low-stress silicon nitride over thermal oxide. The simple process requires four photolithography mask levels. The first defines the interconnect polysilicon layer, while the next two determine the location of the dimple etch and anchor etch in a 2- $-\mu \mathrm{m}$ thick sacrificial oxide layer. The dimples serve as bushings on the structures to reduce sticking and friction when in contact with the substrate. The anchors provide the attachment of the structures to the substrate. The final mask defines the layout of the devices in a 2$\mu \mathrm{m}$ - thick polysilicon layer deposited by Low Pressure Chemical Vapor Deposition (LPCVD) over the sacrificial oxide. At this point, the wafers are immersed in a hydrofluoric-acid-based release etch to produce the free-standing actuators. If necessary, a method such as freeze-sublimation[6] or supercritical carbon dioxide drying [7] could be used to assure the devices are free of final release stiction problems.

\section{DESIGN SUMMARY}

The principle motivation and design goal was to reduce the chip area requirements of linear, in-plane electrostatic actuators such as those used to power the Sandia microengine[8]. A secondary goal was to reduce the sensitivity of the linear actuators to off-axis thrust during operation. The latter is an issue when using the electrostatic comb-drive actuators in the way they are presently utilized in the microengine. The approach we chose was to start with a relatively highenergy-density actuation mechanism such as the electrostatic parallel-plate configuration[9]. The general expression for the electrostatic parallel-plate force is

$\mathrm{F}=\frac{1}{2} \frac{\varepsilon A}{\mathrm{~g}^{2}} \mathrm{~V}^{2}$

where $F$ is the force of attraction between plates, $\varepsilon$ is the permittivity of the gap material, $A$ is the area of the plates, $g$ is the separation gap, and V is the applied voltage across the plates. Many types of microactuators and microsensors make use of this form of electrostatic attraction since it scales very favorably to micron dimensions, i.e., through the inverse-squared gap behavior. Unfortunately, this behavior also makes the force highly non-linear and quite short-ranged. The use of mechanical advantage in the form of levers allows one to minimize these disadvantages by choosing to operate the force over a short-range displacement at a high-force separation.

The design approach was to take this simple idea and implement it in a electro-mechanical, surface micromachined configuration. There are several compromises to be made between optimum mechanical design and process-allowed design. The following list illustrates some of these issues.

Mechanical design issues:

- maximum plate separation,

- plate configurations,

- lever design,

- fulcrum design.

Process issues:

- process minimum dimension,

- minimum plate separation,

- plate configurations,

- fulcrum design.

The easiest manner to discuss the design path is to refer to Figure 1. The primary design shown in Figure 1 exemplifies what we believe to be an optimum compromise between mechanical design and process design. We will start with the configuration of the parallel-plate element. In order to obtain sufficient force with a close-packed configuration, an array of interdigitated fingers is used. One set of fingers belong to the translating linear shuttle element, while two other 
sets form fixed electrodes to the substrate to which the actuation voltage is applied. The primary design constraints on the shuttle are production of the desired output force and the minimization of surface area consumed.

With this information, choices must be made on the number of fingers, length of the fingers, width of the fingers, suitable gap separation between the fixed and movable fingers, range of permissible displacement, and method of attaching the fingers to the drive shuttle. For the length, width, and attachment of the fingers to the shuttle, a maximum allowable deformation of the fingers under electrostatic loading was set at no more than $10 \%$ of the minimum gap separation. This, in turn, determines the total number of fingers required. In an effort to keep the force as constant as possible and to achieve a reasonable level of force, the plates are designed with a $2 \mu \mathrm{m}$ gap in their neutral position with a range of displacement of $1 \mu \mathrm{m}$ giving a minimum gap of $1 \mu \mathrm{m}$. The $2 \mu \mathrm{m}$ neutral, as-fabricated position is well within process capability and the $1 \mu \mathrm{m}$ minimum was set to insure no arcing at 100 volt operation. The minimum gap is set by stops on the shuttle which can be seen in Figure 2.

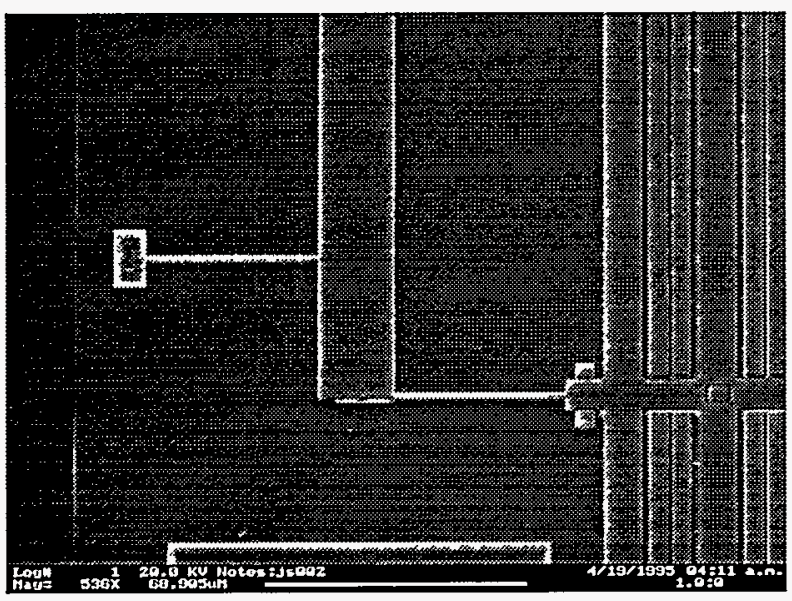

Figure 2 A SEM close-up of the lever, shuttle and stops on the left side of the actuator shown in Figure 1.

Figure 3 illustrates the first lever which comes off the parallel-plate shuttle and the use of flexure element for the fulcrum. The right side of this SEM shows part of the shuttle array. Along the central spine of the shuttle are cutouts with hard stops to the substrate which constrain the length of travel and lateral movement of the shuttle. The shuttle is capable of bidirectional movement constrained to $\pm 1 \mu \mathrm{m}$ by the stops. The sets of two fixed fingers between shuttle fingers have a nominal gap to the shuttle fingers of 2 $\mu \mathrm{m}$ and can have voltage applied to each independently to pull in either direction.

Two basic designs for the shuttle and finger attachment are considered. The simplest is a shuttle with a central spine from which the fingers extend to both sides. The main problem with that design is that the fingers must be made shorter or stiffer to fit the constraint of maximum deformation under load. An alternate, and our primary shuttle design (see Figure 1), is a box-shaped shuttle wherein there is a central spine but also supports running along both ends of the fingers and connecting to the central spine at the shuttle ends thus the box shape. The fingers then act more like doubly clamped beams, allowing narrower fingers for the given loading. The final constraint that was applied to the above variations is the minimization of area consumed.

To obtain the required large range of motion, several different types of lever systems have been designed. Of primary interest is the design of the lever fulcrums. Due to the small range of motion at the shuttle end of the lever, bearings, which have runout, were not desirable. A possible solution to this problem is to employ flexure elements. Unfortunately, with a single lever, these flexure elements tend to produce retarding moments that are too high. To compromise between these two problems, double lever systems have been employed. On the first lever, flexure elements are primarily used. Compared to a single lever system, the first lever of the dual lever system has a smaller range of rotation. Therefore, the retarding moments from the flexure elements are reduced. On the second lever, bearings could be used since the range of motion is much larger. However, for the primary design, flexure fulcrums were also used on the second lever.

A final comment regarding the design is the use of a pull-pull configuration with the shuttle and levers. The primary design is to operate bi-directionally. The pull-pull configuration eliminates the issue of buckling in the relatively thin load transfer elements. This is accomplished by having two first-level levers connected to the shuttle from both ends as seen in Figure 1.

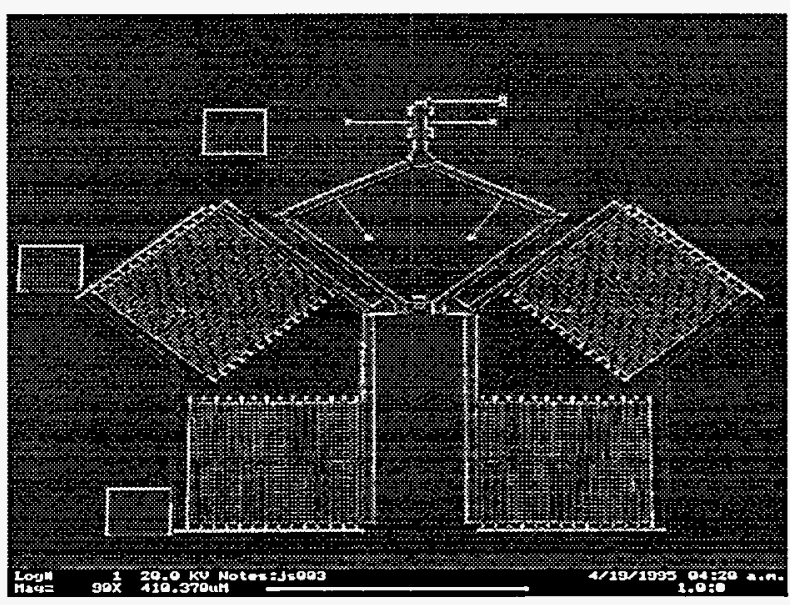

Figure 3 is a SEM of a more elaborate levered device also using the parallel-plate actuator shuttles. The device produces a telescoping action as the actuators are activated. 
Extension of the simple lever configuration to more elaborate designs such as a scissors-action actuator as shown in Figure 3 are also being investigated. This device has been fabricated, but not fully tested.

\section{RESULTS}

Microlevered actuators have been fabricated, released, and tested. The primary design produces actuation force and displacement in very good agreement with the predicted values. The device readily met the overall force and displacement requirements of $\pm 17.5 \mu \mathrm{m}$ and over $2.25 \mu \mathrm{N}$ at 50 Volt operation. Figure 4 is a plot of typical voltagedisplacement for a primary device working against a force-measurement cantilever. At the maximum displacement of $17 \mu \mathrm{m}$ the actuator is sustaining a force of $7.9 \mu \mathrm{N}$.

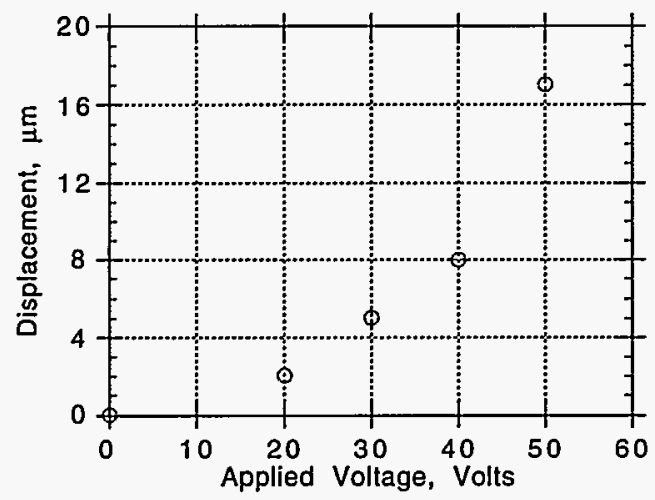

Figure 4 is a plot of displacement versus applied voltage for the microlevered actuator design shown in Figure 1. This displacement is against a cantilever spring element which is producing a restoring force of approximately $7.9 \mu \mathrm{N}$.

\section{CONCLUSION}

The initial results from fabricated microactuators which use mechanical advantage by a system of levers to produce large displacement from a compact array of electrostatic parallel-plate actuators is very promising. The main objective of this approach, to reduce the area consumed by the linear drive actuator element of a microengine, has been accomplished. The reduction in area is roughly by a factor of five, and will be improved in the redesign.

The classical use of levers with fulcrums, when applied to microactuation, appears to work as well at micron dimensions as it does in the macro world. In addition, several other devices with parallel-plate actuation and levers are also being explored for their application as linear or rotary microactuators.

\section{ACKNOWLEDGMENT}

The authors are grateful to several colleagues at Sandia National Laboratories. These include D. Chavez for her invaluable assistance in the fabrication of these devices, $P$. Shea for preparation of SEM micrographs, and the staff of the Microelectronics Development Laboratory at Sandia for supporting the fabrication processes.

\section{REFERENCES}

[1] L. Lin, R. T. Howe, and A. P. Pisano, "A Passive, In Situ Micro Strain Gauge", in Proc. IEEE MicroElectroMechanical Systems, Fort Lauderdale, FL, Feb. 7-10, 1993, pp. 201-206.

[2] C. Keller, and M. Ferrari, "Milli-scale Polysilicon Structures", 1994 Solid-State Sensor and Actuator Workshop, Hilton Head Is., SC, June 133-16, 1994, PP. 132-137.

[3] S. M. Bobbio, M. D. Kellam, B. W. Dudley, S. Goodwin-Johansson, S. K. Jones, J. D. Jacobson, F. M. Tranjan, and T. D. DuBois, "Integrated Force Arrays", in Proc. IEEE MicroElectroMechanical Systems, Fort Lauderdale, FL, Feb. 7-10, 1993, pp. 149-154.

[4] N. Takeshima, K. J. Gabriel, M. Ozaki, J. Takahashi, H. Horiguchi, and H. Fujita, "Electrostatic Parallelogram Actuators", in Proc. 1991 International Conference on Solid-State Sensors and Actuators (TRANSDUCERS '91), San Francisco, CA, June 24-27, 1991, pp. 63-66.

[5] E. J. Garcia and J. J. Sniegowski, "Surface Micromachined Microengine", submitted to Sensors and Actuators.

[6] H. Guckel, J. J. Sniegowski, T. R. Christenson, S. Mohney and T. F. Kelley, "Fabrication of Micromechanical Devices from Polysilicon Films with Smooth Surfaces", Sensors and Actuators, 20 (1989) pp. 117-122.

[7] G. T. Mulhern, D. S. Soane, and R. T. Howe, "Supercritical Carbon Dioxide Drying of Microstructures", in Proc. 1993 International Conference on Solid-State Sensors and Actuators (TRANSDUCERS '93), Yokohama, Japan, June 7-10, 1993, pp. 296-299.

[8] E. J. Garcia and J. J. Sniegowski, "Surface Micromachined Microengine as the Driver for Micromechanical Gears", published in these TRANSDUCERS ' 95 proceedings.

[9] J. J. Sniegowski and E. J. Garcia, "Microfabricated Actuators and Their Application to Optics", SPIE Vol. 2383, Proc. of Miniaturized Systems with Micro-Optics and Micromechanics, Feb. 4-10, 1995, San Jose, CA.

Work performed by Sandia National Laboratories, Albuquerque, NM 87185 for the United States Department of Energy under Contract DE-AC0494AL85000. 